\title{
Nonadiabatic noncyclic geometric phase and persistent current in one-dimensional rings
}

\author{
Z. D. Wang and Shi-Liang Zhu \\ Department of Physics, University of Hong Kong, Pokfulam Road, Hong Kong, People's Republic of China
}

(Received 4 December 1998)

\begin{abstract}
The total geometric phase is composed of the nonadiabatic noncyclic Pancharatnam phase, the usual Aharonov-Bohm $(\mathrm{AB})$ phase, and the effective $\mathrm{AB}$ phase. It is found that the persistent current in onedimensional rings is determined from this phase. As applications, we address first the geometric phase and the persistent current in a ring subject to a cylindrically symmetric electromagnetic field. We show that the Pancharatnam phase recovers the Aharanov-Anandan phase in the case of cyclic evolution, as well as the Berry phase in the adiabatic evolution. Moreover, we discuss the persistent current induced by the spin-orbit-induced geometric phase in the presence of a local magnetic field. Generalization to many-body cases is also addressed. [S0163-1829(99)12239-2]
\end{abstract}

It was discovered by Berry ${ }^{1}$ that a geometric phase $\gamma_{n}(c)=i \oint_{c}\left\langle n(\vec{R})\left|\nabla_{\vec{R}}\right| n(\vec{R})\right\rangle \cdot d \vec{R}$, in addition to the usual dynamical phase, $-1 / \hbar \int_{0}^{\tau} E_{n}(\vec{R}(t)) d t$, is accumulated on the wave function of a quantum system, provided that the Hamiltonian is cyclic and adiabatic. This adiabatic geometric phase, commonly referred to as the Berry phase, has found many applications in condensed matter physics ${ }^{2,3}$. A further generalization of Berry's concept was introduced by Aharonov and Ananda (AA), ${ }^{4}$ provided that the evolution of the state is cyclic. Besides, Samuel and Bhandari (SB) introduced a more general geometric phase in the nonadiabatic noncyclic evolution of the system (the Pancharatnam phase). ${ }^{5}$ The noncyclic evolution is quite ordinary in a quantum system, however, the SB's generalization has so far appeared to be merely a mathematical point of view, and few realistic systems have been addressed by using this generalization.

On the other hand, persistent currents (PC) in mesoscopic normal metal rings have recently drawn considerable interests. ${ }^{6-8}$ Many authors ${ }^{9-15}$ addressed the geometric phase in such mesoscopic systems. Loss et al. investigated the adiabatic electronic transport in mesoscopic rings embedded in a magnetic field, and found that the persistent charge and spin currents can be induced by the Berry phase. ${ }^{9}$ The PC induced by geometric Aharonov-Casher (AC) effect from the symmetric electric field was proposed. ${ }^{10,11}$ The PC of certain hard-core bosons induced by AC effect was addressed in Ref. 12. Noticing these investigations, a more fundamental and important question arises: can certain type of PC exist so long as the geometric phase is non-zero? On the other hand, the adiabatic approximation has been widely used in many theoretical studies $9,11,13,14$ though the adiabatic approximation is not applicable in mesoscopic rings in many cases. The adiabatic condition $\mu B a \gg \hbar V_{f}$ has been obtained by Stern $^{13}$ for a ring subject to a magnetic field $B$, with the ring radius as $a$, the electron magnetic moment as $\mu$, and the Fermi velocity as $V_{f}$. For copper rings considered in Ref. 7, $V_{f}$ $\sim 1.57 \times 10^{5} \mathrm{~m} / \mathrm{s}, a \sim 2.3 \times 10^{-6} \mathrm{~m}, B \sim 10^{-2}$ Tesla. For GaAsAlGaAs single loop considered in Ref. 8, $V_{f} \sim 2.6$ $\times 10^{5} \mathrm{~m} / \mathrm{s}, a \sim 8.4 \times 10^{-6} \mathrm{~m}, B \sim 10^{-2}$ Tesla. In those two typical mesoscopic systems, the adiabatic condition is not satisfied. Furthermore, in general, a quasiparticle wave function in a mesoscopic ring may follow a noncyclic evolution, and thus further theoretical understanding of noncyclic evolution is greatly desirable. However, as we know, general noncyclic cases have not yet been addressed in the study of the geometric phase in mesoscopic systems though the properties of noncyclic evolution may be abundant.

In this paper, we address the nonadiabatic and noncyclic geometric phase in mesoscopic rings, the sizes of which are less than the phase relaxation length of quantum particles such that the quantum mechanics can be used directly. We demonstrate that persistent probability currents are determined from the total geometric phase. As applications, we consider first mesoscopic rings subject to crown-shaped magnetic and electric fields both with constant tilting angles. Secondly, we address PC induced by the spin-orbit(SO)induced geometric phase in the presence of local magnetic field. Finally, we generalize our formulation to many-body cases.

Let us first introduce briefly the generalization of Berry phase to a nonadiabatic noncyclic evolution. ${ }^{5,16}$ For a quantum system whose normalized dimensionless state vector $|\psi\rangle$ (an element of a Hilbert space $H$ ) evolves according to the Schrödinger equation $i \hbar \partial / \partial t|\psi(t)\rangle=\hat{H}|\psi(t)\rangle$, the phase accumulated on an evolution of $|\psi(s)\rangle$ (with $s$ as a parameter) from $s_{1}$ to $s_{2}$ (along a geodesic path $1 \rightarrow 2$ ) is given by a generalized Pancharatnam phase

$$
\tilde{\gamma}_{S B}=\operatorname{Im} \int_{1 \rightarrow 2}\langle\psi|d / d s| \psi\rangle d s .
$$

If the dynamical phase can be eliminated from $\tilde{\gamma}_{S B}$, it becomes the standard Pancharatnam phase $\gamma_{S B} \cdot{ }^{5,16}$

We here consider a particle (e.g., an electron) with mass $m$ confined to a mesoscopic ring (with the circumference $L_{c}$, radius $a$ ) subject to electromagnetic fields. Such a mesocsopic ring may be fabricated on the basis of a highly mobile two-dimensional electron gas. For brevity, we denote $\mathbf{A}^{\mathrm{em}}$ $=e \mathbf{A}-\mu \mathbf{a}$ as an effective electromagnetic vector potential of the spin- $\frac{1}{2}$ system, where $\mathbf{A}$ is the usual $U(1)$ vector potential in connection with charge $-e$ and $\mathbf{a}$ is an effective $S U(2)_{\text {spin }}$ spin-dependent vector potential in connection 
with magnetic magneton $\mu$ [see, e.g., the detail below Eq. (7)]. In the cylindrical coordinate $(r, \varphi, z)$, the onedimensional current density operator in the ring is given by $\hat{j}=-i\left(\hbar / m L_{c}\right)(\partial / \partial \varphi)-\left(1 / m L_{c}\right) \mathbf{A}^{e m}$, and thus the current carried by an arbitrary state $|\psi\rangle$ in the ring is found to be

$$
\begin{array}{r}
j=\frac{\hbar}{m L_{c}^{2}}\left[\operatorname{Im} \int_{0}^{2 \pi} d \varphi\langle\psi|d / d \varphi| \psi\rangle\right. \\
\left.-\frac{1}{\hbar} \int_{0}^{2 \pi} d \varphi\left\langle\psi\left|\frac{L_{c}}{2 \pi} A_{\varphi}^{e m}\right| \psi\right\rangle\right],
\end{array}
$$

where $A_{\varphi}^{e m}$ is the $\varphi$ component of $\mathbf{A}^{e m}$. It is of interest to note that the first term in the brackets of the right-hand-side (RHS) of Eq. (2) is just the generalized Pancharatnam phase accumulated on the evolution when the parameter $\varphi$ changes from 0 to $2 \pi$, and the second term represents the total Aharonov-Bohm (AB) phase $\gamma_{A B}^{T o t}=$ - $(1 / \hbar) \int_{0}^{2 \pi} d \varphi\left\langle\psi\left|\left(L_{c} / 2 \pi\right) A_{\varphi}^{e m}\right| \psi\right\rangle$, which includes the usual AB phase $\gamma_{A B}$ and the effective AB phase $\gamma_{A B}^{\text {eff }}$ (see the later discussion for details). The total phase accumulated on the evolution can then be defined as $\delta \gamma=\tilde{\gamma}_{S B}+\gamma_{A B}^{T o t}$. Therefore, we can rewrite Eq. (2) as

$$
j=\frac{\hbar}{m L_{C}^{2}} \delta \gamma
$$

It is worth pointing out that the macroscopically observable current is the statistically averaged current $\langle j\rangle$ $=\left(\hbar / m L_{C}^{2}\right)\langle\delta \gamma\rangle$. Practically, the above formula can be further simplified in many physical systems including the models we will discuss below.

If the $\mathbf{A}^{e m}$ is independent of the time explicitly, so is the Hamiltonian $\hat{H}$ of the ring (see, e.g., Eqs. (7) and (13) below). The stationary Schrödinger equation is $\hat{H}|l(\varphi)\rangle$ $=\varepsilon_{l}|l(\varphi)\rangle$ with $\varepsilon_{l}$ being time-independent eigen-energy and $|l(\varphi)\rangle$ eigen-function, where $(1 / 2 \pi) \int_{0}^{2 \pi}\langle l(\varphi) \mid k(\varphi)\rangle d \varphi$ $=\delta_{l k}$. A dimensionless state vector can be expressed as $|\psi(t)\rangle=\Sigma_{l} a_{l} \exp \left(-i \varepsilon_{l} t / \hbar\right)|l(\varphi)\rangle, \quad$ where $\quad a_{l}$ $=(1 / 2 \pi) \int_{0}^{2 \pi}\langle l(\varphi) \mid \psi(0)\rangle d \varphi$ is time-independent. If the condition $\int_{l \neq k}\langle l(\varphi)|d / d \varphi| k(\varphi)\rangle d \varphi=0$ is satisfied, ${ }^{17} \tilde{\gamma}_{S B}$ may further be written as

$$
\tilde{\gamma}_{S B}=\operatorname{Im} \int_{0}^{2 \pi} \sum_{l} a_{l}^{*} a_{l}\left\langle l(\varphi)\left|\frac{d}{d \varphi}\right| l(\varphi)\right\rangle d \varphi=\gamma_{S B},
$$

where the dynamic phase has been canceled. Although Eq. (4) is similar to the definition of the Berry phase except that $\gamma_{S B}$ takes the averaged value, it should be emphasized that Eq. (4) can be used in a nonadiabatic noncyclic evolution. Of course, $-\gamma_{S B}$ becomes the Berry phase for an adiabatic evolution of any eigenstate of $\hat{H}$. Moreover, the total AB phase may be rewritten as

$$
\gamma_{A B}^{T o t}=-\frac{1}{\hbar} \int_{0}^{2 \pi} d \varphi \sum_{l} a_{l}^{*} a_{l}\left\langle l(\varphi)\left|\frac{L_{c}}{2 \pi} A_{\varphi}^{e m}\right| l(\varphi)\right\rangle .
$$

We now have the relation $d j / d t=0$, which implies that $j$ is an equilibrium persistent probability current $j^{p}$ and is determined from the total geometric phase $\delta \gamma_{g}=\gamma_{S B}+\gamma_{A B}^{T o t}$, i.e.,

$$
j^{p}=\frac{\hbar}{m L_{C}^{2}} \delta \gamma_{g}
$$

For an equilibrium system, the calculation of the observable persistent current $\left\langle j^{p}\right\rangle$ should be subject to the constrain that the free energy of the system is minimized.

The persistent probability currents can be displayed in different ways and some of them may be observed in experiments. For examples, (i) for the persistent charge current $I$ $=-e\left\langle j^{p}\right\rangle$ carried by $N$ non-interacting electrons in a mesoscopic normal-metal ring subject to an $\mathrm{AB}$ flux, ${ }^{6-8}$ we have $I=\left(-e \hbar / m L_{c}^{2}\right) \sum_{r=1}^{N}\left\langle\delta \gamma_{g}^{(r)}\right\rangle$, where $\delta \gamma_{g}^{(r)}$ is the geometric phase of the $r$-th particle in the ring; (ii) the persistent mass and spin currents can be induced by the AC effect; ${ }^{10,11}$ (iii) the persistent fluxon currents may be carried by neutral hardcore bosons with magnetic moments. ${ }^{12}$

$\mathrm{PC}$ in mesoscopic rings reflects the broken clockwiseanticlockwise symmetry of charge carriers momenta. Ihm demonstrated that in the absence of singularity, Berry phase aries only if the time-reversal symmetry is broken. ${ }^{18}$ The time-reversal symmetry implies that the same phase factor, including both geometric and dynamical phases, would be accumulated in the evolution of an electron moving around the ring in both clockwise and anticlockwise directions. However, notice that the geometric phases accumulated in the two directions of evolution have different signs even though two dynamical phases are identical. Therefore, a kind of PC can be induced by the nonzero geometric phase associated with the broken time-reversal symmetry in mesoscopic rings. As applications of Eq. (6), we investigate first the PC and the geometric phase induced by electromagneic fields, and then address a novel kind of PC induced by the $\mathrm{SO}$-induced geometric phase in the presence of a local magnetic field.

The nonrelativistic Hamiltonian with $U(1)_{\mathrm{em}}$ $\times S U(2)_{\text {spin }}$ gauge symmetry of an electron with charge $-e$ and spin $1 / 2$ in an external electromagnetic field is given by $^{10}$

$$
\hat{H}=\frac{1}{2 m}(\mathbf{p}+e \mathbf{A}-\mu \mathbf{a})^{2}-e A^{0}+\mu a^{0},
$$

where $A^{\nu}=\left(A^{0}, \mathbf{A}\right)$ represent four $U(1)_{e m}$ electromagnetic vector potentials, and $a^{\nu}=\left(a^{0}, \mathbf{a}\right)=(-\vec{\sigma} \cdot \mathbf{B}, \vec{\sigma} \times \mathbf{E} / 2)$ is an $S U(2)_{\text {spin }}$ potential with $\vec{\sigma}$ as the vector of Pauli matrices. Clearly, the $S U(2)_{\text {spin }}$ vector potential a plays a similar role to that of the $U(1)_{e m}$ vector potential $\mathbf{A}$ in the $\mathrm{AB}$ effect. Therefore, the effective vector potential may be defined as $\mathbf{A}^{e m}=e \mathbf{A}-\mu \mathbf{a}$. Thus one can expect that an effective $\mathrm{AB}$ phase can be induced by this $S U(2)_{\text {spin }}$ vector potential. The Hamiltonian of the ring, subject to cylindrically symmetric electromagnetic fields $\mathbf{E}(\varphi)=E\left(\sin \chi \mathbf{e}_{r}+\cos \chi \mathbf{e}_{z}\right), \mathbf{B}(\varphi)$ $=B\left(\sin \theta \mathbf{e}_{r}+\cos \theta \mathbf{e}_{z}\right)$, and a magnetic flux $\phi$, is given by

$$
\begin{aligned}
\hat{H}= & \hbar \omega\left[-i \frac{d}{d \varphi}+\frac{\phi}{\phi_{0}}-\frac{\eta}{2}\left(\cos \chi \cos \varphi \sigma_{x}+\cos \chi \sin \varphi \sigma_{y}\right.\right. \\
& \left.\left.-\sin \chi \sigma_{z}\right)\right]^{2}-\mu B\left(\sin \theta \cos \varphi \sigma_{x}+\sin \theta \sin \varphi \sigma_{y}\right. \\
& \left.+\cos \theta \sigma_{z}\right),
\end{aligned}
$$


where $\omega=\hbar / 2 m a^{2}, \phi_{0}=2 \pi \hbar / e, \eta=\mu E a / \hbar$ is the normalized electric field strength, and $\theta(\chi)$ is the tilting angle between the magnetic(electric) field and $z$ axis. This Hamiltonian can be diagonalized as

$$
\hat{H}=\sum_{n \alpha} E_{n \alpha} \hat{a}_{n \alpha}^{+} \hat{a}_{n \alpha}, \quad(\alpha= \pm)
$$

with

$$
E_{n \alpha}=\frac{\varepsilon_{n+}+\varepsilon_{(n+1)-}}{2}+\alpha \sqrt{g_{n}^{2}+\triangle_{n}^{2}},
$$

where

$$
\begin{gathered}
\varepsilon_{n \pm}=\hbar \omega\left(n+\frac{\phi}{\phi_{0}} \pm \frac{1}{2} \eta \sin \chi\right)^{2} \mp \mu B \cos \theta+\frac{\hbar \omega}{4} \eta^{2} \cos ^{2} \chi, \\
\triangle_{n}=\mu B \sin \theta+\hbar \omega \eta \cos \chi\left(n+\frac{1}{2}+\frac{\phi}{\phi_{0}}\right),
\end{gathered}
$$

and $g_{n}=\left(\varepsilon_{(n+1)-}-\varepsilon_{n+}\right) / 2, n=0, \pm 1, \pm 2, \ldots$ The eigenfunctions are given by

$$
\left|\xi_{n+(\varphi)}\right\rangle=\exp (i n \varphi)\left(\begin{array}{c}
\cos \left(\beta_{n} / 2\right) \\
e^{i \varphi} \sin \left(\beta_{n} / 2\right)
\end{array}\right)
$$

and

$$
\left|\xi_{n-(\varphi)}\right\rangle=\exp (i n \varphi)\left(\begin{array}{c}
-e^{-i \varphi} \sin \left(\beta_{n} / 2\right) \\
\cos \left(\beta_{n} / 2\right)
\end{array}\right),
$$

with $\operatorname{tg} \beta_{n}=\triangle_{n} / g_{n}$. Clearly, in the $\left|\xi_{n \alpha}(\varphi)\right\rangle$ state the spin aligns with the angle $\beta_{n \alpha}$ to the z-axis. An arbitrary state $|\psi(\varphi, t)\rangle=\Sigma_{n \alpha} a_{n \alpha} \exp \left(-i E_{n \alpha} t / \hbar\right)\left|\xi_{n \alpha}(\varphi)\right\rangle$ may not take a cyclic evolution because the spectrum $E_{n \alpha}$ does not satisfy the cyclic condition, ${ }^{19}$ such that only the Pancharatnam phase is relevant. Since $\int_{n \alpha \neq n^{\prime} \alpha^{\prime}} d \varphi\left\langle\xi_{n \alpha}(\varphi)|d / d \varphi| \xi_{n^{\prime} \alpha^{\prime}}(\varphi)\right\rangle=0$, we can employ Eq. (4) to evaluate the Pancharatnam phase

$$
\gamma_{S B}=2 \pi \sum_{n \alpha} n a_{n \alpha}^{*} a_{n \alpha}+\alpha \pi\left(1-\sum_{n \alpha} a_{n \alpha}^{*} a_{n \alpha} \cos \beta_{n}\right) .
$$

Meanwhile, the total $\mathrm{AB}$ phase is given by

$$
\begin{aligned}
\gamma_{A B}^{T o t}= & \frac{e}{\hbar} \int_{0}^{2 \pi} d \varphi \sum_{n \alpha} a_{n \alpha}^{*} a_{n \alpha}\left\langle\xi_{n \alpha}(\varphi)\left|\frac{L_{c}}{2 \pi} A_{\varphi}\right| \xi_{n \alpha}(\varphi)\right\rangle \\
& -\frac{\mu}{\hbar} \int_{0}^{2 \pi} d \varphi \sum_{n \alpha} a_{n \alpha}^{*} a_{n \alpha}\left\langle\xi_{n \alpha}(\varphi)\left|\frac{L_{c}}{2 \pi} a_{\varphi}\right| \xi_{n \alpha}(\varphi)\right\rangle \\
= & \gamma_{A B}+\gamma_{A B}^{e f f}
\end{aligned}
$$

where $A_{\varphi}=\phi / L_{c}$ and $a_{\varphi}=E / 2\left(\cos \chi \cos \varphi \sigma_{x}+\cos \chi \sin \varphi \sigma_{y}\right.$ $\left.-\sin \chi \sigma_{z}\right)$. It is straightforward to find that $\gamma_{A B}=2 \pi \phi / \phi_{0}$ is the usual $\mathrm{AB}$ phase induced by the magnetic flux and $\gamma_{A B}^{\text {eff }}$ $=-\pi \eta \Sigma_{n \alpha} \alpha a_{n \alpha}^{*} a_{n \alpha} \sin \left(\beta_{n}-\chi\right)$ is an effective AB phase induced by the crown-shaped electromagnetic field. In the special case of $B=0$, Choi et al. found that the total geometric phase induced by the electric field is the sum of the effective $\mathrm{AB}$ phase and the AA phase when the cyclic condition is satisfied. ${ }^{15}$ Actually, we here find that the total geometric phase in a mesoscopic ring is composed of the usual $A B$ phase, the effective $\mathrm{AB}$ phase, and the Pancharatnam phase in the case of noncyclic evolution.

To compare our results with the previous results conveniently, we now discuss the geometric phase in the case of $\eta=0$ and $B \neq 0$. The AA phase exists when $(n, \alpha)$ is chosen to be a specific $\left(n_{0}, \alpha_{0}\right)$, because the system is in a cyclic state in this case. ${ }^{20}$ If we denote the Pancharatnam phase as $\gamma_{S B}^{n_{0} \alpha_{0}}$ when $a_{n \alpha}=\delta_{n n_{0}} \delta_{\alpha \alpha_{0}}$, Eq. (10) leads to $\gamma_{S B}^{n_{0} \alpha_{0}}=2 n_{0} \pi$ $+\alpha_{0} \pi\left(1-\cos \beta_{n_{0}}\right)$. This result is similar to example two in Ref. 4 except that here the angle $\beta_{n_{0}}$ links with the quantum number $n_{0}$. Using the expression of $\left|\xi_{n_{0} \alpha_{0}}(\varphi)\right\rangle$, we can find that the AA phase is the same as $\gamma_{S B}^{n_{0} \alpha_{0}}$. Therefore, we have examined that $\gamma_{A A}=\gamma_{S B}$ when the system takes a cyclic evolution. Under the adiabatic condition, $\beta_{n_{0}}=\theta, \alpha_{0}=\sigma_{0}$ (the index of spin), and $-\gamma_{S B}^{n_{0} \alpha_{0}}$ just gives out the Berry phase $\gamma_{\text {Berry }}^{n_{0} \sigma_{0}} \cdot 9,13,14$

As a typical example, the statistically averaged PC in the ring with $N$ non-interacting electrons can be represented as

$$
\begin{aligned}
\bar{I}= & -\frac{e \hbar}{m L_{C}^{2}} \sum_{r}\left\langle\delta \gamma_{g}^{(r)}\right\rangle=-\frac{e \hbar}{2 \pi m a^{2}} \sum_{n \alpha}\left[n+\frac{\phi}{\phi_{0}}\right. \\
& \left.+\alpha \sin ^{2}\left(\beta_{n} / 2\right)-\alpha \frac{\eta}{2} \sin \left(\beta_{n}-\chi\right)\right] \overline{a_{n \alpha}^{*} a_{n \alpha} .}
\end{aligned}
$$

For $N$ non-interacting particles which obey Fermi-Dirac distribution $f\left(E_{n \alpha}\right), \overline{a_{n \alpha}^{*} a_{n \alpha}}=f\left(E_{n \alpha}\right)$. Of course, in the case of $E=0$ and $B=0$, Eq. (12) reduces to the well-known result $\bar{I}=-\left(e \hbar / \pi m a^{2}\right) \Sigma_{n}\left[n+\left(\phi / \phi_{0}\right)\right] f\left(E_{n}\right)$.

Another interesting application is PC induced by the SOinduced geometric phase in the presence of a local magnetic field. The electron spin in a low dimensional structure is influenced by the momentum-dependent effective magnetic field because the electron Hamiltonian includes a term linear in momentum $P$, i.e., $\hat{H}=\left(P^{2} / 2 m\right)+(\hbar / 2) \sum_{i, j} \sigma_{i} \beta_{i j} P_{j} \quad\left(\beta_{i j}\right.$ is the SO splitting coefficient), which describes the SO splitting of the electron states at $P \neq 0$. This SO interaction leads to geometric phase effects in conducting rings. ${ }^{14}$ Thus, $\mathrm{PC}$ in the presence of an external (or internal ${ }^{21}$ ) local field can be induced by this geometric phase. For a one-dimensional ring subject to a local magnetic field $B_{L} \vec{e}_{z}$, the Hamiltonian in the cylindrical coordinates is given by ${ }^{22}$

$$
\begin{aligned}
\hat{H}= & \hbar \omega\left(-i \frac{\partial}{\partial \varphi}+\frac{\phi}{\phi_{0}}\right)^{2}+\hbar \omega_{1}\left(\sigma_{x} \cos \varphi+\sigma_{y} \sin \varphi\right) \\
& \times\left(-i \frac{\partial}{\partial \varphi}+\frac{\phi}{\phi_{0}}\right)+\frac{i \hbar \omega_{1}}{2}\left(\sigma_{x} \sin \varphi-\sigma_{y} \cos \varphi\right) \\
& +\hbar \omega_{2} \sigma_{z}\left(-i \frac{\partial}{\partial \varphi}+\frac{\phi}{\phi_{0}}\right)-\mu B_{L} \sigma_{z},
\end{aligned}
$$

where $\omega_{i}=\hbar \beta_{i} / 2 a$. Equation (13) may be rewritten as

$$
\hat{H}=\hbar \omega\left[-i \frac{\partial}{\partial \varphi}+\frac{\phi}{\phi_{0}}-\frac{\mu}{a} a_{\varphi}\right]^{2}-\mu B_{L} \sigma_{z}-\frac{\omega_{1}^{2}+\omega_{2}^{2}}{4 \omega^{2}},
$$


where $a_{\varphi}=(-a / 2 \omega \mu)\left[\omega_{1}\left(\sigma_{x} \cos \varphi+\sigma_{y} \sin \varphi\right)+\omega_{2} \sigma_{z}\right]$ is the $\varphi$-component of the spin-dependent vector potential. The energy spectrum can be obtained as

$$
E_{n \alpha}^{S O}=\frac{\hbar \omega}{2}\left[\left(n+\frac{\phi}{\phi_{0}}\right)^{2}+\left(n+1+\frac{\phi}{\phi_{0}}\right)^{2}\right]+\alpha \sqrt{\nu_{n}^{2}+\lambda_{n}^{2}},
$$

with

$$
\nu_{n}=\hbar\left(\omega-\omega_{2}\right)\left(n+\frac{1}{2}+\frac{\phi}{\phi_{0}}\right)+\mu B_{L},
$$

and

$$
\lambda_{n}=\hbar \omega_{1}\left(n+\frac{1}{2}+\frac{\phi}{\phi_{0}}\right) .
$$

The statistically averaged $\mathrm{PC}$ of $\mathrm{N}$ non-interacting electrons is found to be

$$
\bar{I}=-\frac{e \hbar}{2 \pi m a^{2}} \sum_{n \alpha}\left[n+\frac{\phi}{\phi_{0}}+\frac{\phi_{\alpha}^{S O}}{\phi_{0}}\right] \overline{a_{n \alpha}^{*} a_{n \alpha}},
$$

where $\quad \phi_{\alpha}^{S O}=\phi_{0} / 2\left\{1+(\alpha / \omega)\left[\left(\omega-\omega_{2}\right) \nu_{n}+\omega_{1} \lambda_{n}\right] /\right.$ $\left.\sqrt{\nu_{n}^{2}+\lambda_{n}^{2}}\right\}$. We wish to make a few remarks on this model. (i) In the case of $\phi=B_{L}=0$, the averaged PC should be zero because the energy $E_{n \alpha}$ is exactly equal to $E_{-(n+1) \alpha} \cdot{ }^{23}$ (ii) The SO-induced geometric phase contributes to the PC, provided that $B_{L} \neq 0$ or $\phi \neq 0$. (iii) It was reported very recently that the SO-induced Berry phase effect had been observed experimentally. ${ }^{24} \mathrm{We}$ thus expect that this kind of PC may be observable in a similar mesoscopic experiment. Estimation ${ }^{14}$ indicates that, for an InAs ring with radius as $5 \mu \mathrm{m}$ and width as $60 \mathrm{~nm}, m \sim 0.023 m_{0}, \phi_{\alpha}^{S O} / \phi_{0} \sim 6$, which may induce an observable effect.

Finally, we may generalize our discussion to many-body cases. The Schrödinger equation for $\mathrm{N}$ particles in a ring reads $i \hbar(\partial / \partial t)\left|\Psi_{\left(\varphi_{1} \cdots \varphi_{N}\right)}\right\rangle=\hat{H}\left|\Psi_{\left(\varphi_{1} \cdots \varphi_{N}\right)}\right\rangle$. The manyparticle quantum phase may be expressed as

$$
\begin{aligned}
\delta \gamma(N)= & \frac{1}{(2 \pi)^{N-1}} \operatorname{Re} \int_{0}^{2 \pi} d \varphi_{1} \ldots \int_{0}^{2 \pi} d \varphi_{N}\left\langle\Psi_{\left(\varphi_{1} \cdots \varphi_{N}\right)}\right| \\
& \times \sum_{r=1}^{N}\left[-i \frac{d}{d \varphi_{r}}-\frac{L_{c}}{2 \pi \hbar} A_{\varphi_{r}}^{e m}\right]\left|\Psi_{\left(\varphi_{1} \cdots \varphi_{N}\right)}\right\rangle .
\end{aligned}
$$

As a result, we still have $\langle j\rangle=\left(\hbar / m L_{C}^{2}\right)\langle\delta \gamma(N)\rangle$. For $N$ non-interacting particles, it is not difficult to derive that $\delta \gamma(N)=\sum_{r=1}^{N} \delta \gamma^{(r)}$. Clearly, the statistically averaged current $\bar{I}$ obtained before can be naturally recovered. We wish to remark that the above generalization may allow us to include the effect of electron-electron interaction, which is highly nontrivial and may be investigated in the future.

This work was supported by a CRCG grant at the University of Hong Kong.
${ }^{1}$ M.V. Berry, Proc. R. Soc. London, Ser. A 392, 45 (1984).

${ }^{2}$ J.E. Avron, Phys. Rev. Lett. 75, 697 (1995).

${ }^{3}$ Q. Niu and L. Kleinman, Phys. Rev. Lett. 80, 2205 (1998).

${ }^{4}$ Y. Aharonov and J. Anandan, Phys. Rev. Lett. 58, 1593 (1987).

${ }^{5}$ J. Samuel and R. Bhandari, Phys. Rev. Lett. 60, 2339 (1988).

${ }^{6}$ M. Buttiker, Y. Imry, and R. Landauer, Phys. Lett. A96, 365

(1983); H.F. Cheung, Y. Gefen, E.K. Riedel, and W.-H. Shih, Phys. Rev. B 37, 6050 (1988); Q. Wang et al., ibid. 54, 8101 (1996).

${ }^{7}$ L.P. Levy, G. Dolan, J. Dunsmuir, and Bouchiat, Phys. Rev. Lett. 64, 2074 (1990).

${ }^{8}$ D. Mail, C. Chapelier, and A. Benoit, Phys. Rev. Lett. 70, 2020 (1993).

${ }^{9}$ D. Loss, P. Goldbart, and A.V. Balatsdy, Phys. Rev. Lett. 65, 1655 (1990); D. Loss and P. Goldbart, Phys. Rev. B 45, 13544 (1992).

${ }^{10}$ S. Oh and C.-M. Ryu, Phys. Rev. B 51, 13441 (1995).

${ }^{11}$ A.V. Balatsky and B.L. Altshuler, Phys. Rev. Lett. 70, 1678 (1993).

${ }^{12}$ Z.D. Wang and Jian-Xin Zhu, Phys. Rev. B 52, 5275 (1995); Jian-Xin Zhu, Z.D. Wang, and L. Sheng, ibid. 52, 14505 (1995); Jian-Xin Zhu and Z. D. Wang, Phys. Rev. A 53, 600 (1996).

${ }^{13}$ A. Stern, Phys. Rev. Lett. 68, 1022 (1992).

${ }^{14}$ A.G. Aronov and Y. Lyanda-Geller, Phys. Rev. Lett. 70, 343 (1993); Y. Lyanda-Geller, ibid. 71, 657 (1993).

${ }^{15}$ T. Choi, S.Y. Cho, C.-M. Ryu, and C.K. Kim, Phys. Rev. B 56, 4825 (1997).

${ }^{16}$ Y.S. Wu and H.Z. Li, Phys. Rev. B 38, 11907 (1988).
${ }^{17}$ The wave function in a ring satisfies the relation $|\psi(\varphi)\rangle=\mid \psi(\varphi$ $+2 \pi)\rangle$, we therefore have $|l(\varphi)\rangle=e^{i l \varphi} \hat{f}_{l}(\varphi)$ according to the Bloch theorem. As a result, $\int_{0}^{2 \pi}\langle l(\varphi)|d / d \varphi| k(\varphi)\rangle d \varphi$ $=\int_{0}^{2 \pi} e^{i(k-l) \varphi} \hat{f}_{l}^{+}(\varphi)(d / d \varphi) \hat{f}_{k}(\varphi) d \varphi=0$ for $l \neq k$, if ever $d / d \varphi\left[\hat{f}_{l}^{+}(\varphi)(d / d \varphi) \hat{f}_{k}(\varphi)\right]=0$, which is satisfied in the models addressed in the present paper.

${ }^{18}$ J. Ihm, Phys. Rev. Lett. 67, 251 (1991).

${ }^{19}$ The necessary and sufficient condition for the existence of cyclic evolution for an arbitrary state in a closed system is that the spectrum of the system can be represented as $\varepsilon_{l}=\varepsilon_{0}+m_{l} \hbar \omega_{0}$, where $m_{l}$ is an integer, $\omega_{0}>0$, and $\varepsilon_{0}$ is real. See A.N. Seleanyova, J. Phys. A 26, 981 (1993).

${ }^{20}$ Some specific states superposed linearly by no more than two eigenstates may follow a cyclic evolution regardless of the spectrum.

${ }^{21}$ I.D. Vagner, A.S. Rozhavsky, P. Wyder, and A.Yu. Zyuzin, Phys. Rev. Lett. 80, 2417 (1998).

${ }^{22}$ Yi-Chang Zhou, Hua-Zhong Li, and Xun Xue, Phys. Rev. B 49, 14010 (1994).

${ }^{23}$ In the case of $\phi=B_{L}=0$, the energy Eq. (14) can be written as

$$
E_{n \alpha}^{S O}=\hbar \omega\left(n+\frac{1}{2}\right)^{2}+\alpha\left|n+\frac{1}{2}\right| \sqrt{\left(\omega-\omega_{2}\right)^{2}+\omega_{1}^{2}}+\frac{1}{4} \hbar \omega .
$$

It is clear that $E_{n \alpha}=E_{-(n+1) \alpha}$. Meanwhile Eq. (15) becomes

$$
\bar{I}=-\frac{e \hbar}{2 \pi m a^{2}} \sum_{n \alpha}\left[n+\frac{1}{2}+\frac{\alpha \hbar}{2 \omega} \sqrt{\left(\omega-\omega_{2}\right)^{2}+\omega_{1}^{2}}\right] \overline{a_{n \alpha}^{*} a_{n \alpha}} .
$$

Therefore the currents carried by electrons in the completely filled levels $E_{n}$ and $E_{-(n+1)}$ should be canceled.

${ }^{24}$ A.F. Morpurgo, J.P. Heida, T.M. Klapwijk, B.J. Van Wees, and G. Borghs, Phys. Rev. Lett. 80, 1050 (1998). 